\title{
Ultrasonic measurements of surface defects on flexible circuits using high frequency focused polymer transducers
}

\author{
Sanat Wagle*, Anowarul Habib, and Frank Melands $\varnothing^{*}$ \\ Department of Physics and Technology, UiT The Arctic University of Norway, \\ 9037 Troms $\varnothing$, Norway
}

\section{Corresponding author}

*Frank Melandsø, Sanat Wagle

Department of Physics and Technology, UiT The Arctic University of Norway, Troms $\varnothing$,

Norway

Tel: +47-77645666

Fax: $+47-77645580$

E-mail address: frank.melandso@uit.no,sanat.wagle@uit.no 


\begin{abstract}
High frequency transducers made from a layer-by-layer deposition method are investigated as transducers for ultrasonic imaging. Prototypes of adhesive-free transducers with four active elements were made on a high performance PEI (Polyetherimide) substrate with precision milled spherical cavities used to produce focused ultrasonic beams. The transducer prototypes were characterized using a pulse-echo experimental setup in water tank using glass plate as a reflector. Then, it was used in a 3D ultrasonic scanning tank, to produce high-resolution ultrasonic images of flexible electronic circuits with the aim to detect defects in the outermost cover layer.
\end{abstract}


Ferroelectric polyvinylidenefluoride (PVDF) and its copolymer PVDF trifluoroethylene [P(VDF-TrFE)] can be processed by a number of methods, and have low acoustic impedance compared to ceramics, which matches well with water and human tissue. ${ }^{1-4)}$ Hence, they are widely used for making ultrasonic sensors, transducers, hydrophones and actuators for different application such as biomedical imaging, and nondestructive evaluation of material. ${ }^{1-4)}$ Although, these piezoelectric polymers and co-polymers demerit from higher attenuation and lower electromechanical coupling properties compare to ceramics, the simplified material processing and low impedance make polymer-based transducer attractive, especially at high frequencies. ${ }^{4)}$ Recently, we have also shown that the P(VDF-TrFE) can be screen-printed and spin coated to produce high frequency single element and array ultrasonic transducers with Polyetherimide (PEI) as the backing substrate. ${ }^{5-8)}$

For high frequency focused ceramic or crystal transducers, the acoustic energy is typically focused using a concave spherical sapphire lens rod. They are often used for better image resolution or noninvasive micro-structural characterization of materials, characterization of surface and subsurface mechanical properties of piezoelectric materials. $\left.{ }^{9}, 10\right)$ The main disadvantage with such a design is the impedance mismatch between the refractive lens material (e.g., sapphire) and water, yielding for example, reduced sound transmission, bandwidth reduction, and geometrical aberration of the focusing beam. ${ }^{11-13)}$ Some works also employed pre-poled flexible piezoelectric polymer film to fabricate focused transducers ${ }^{1,2,11-}$ ${ }^{13)}$ where additional adhesive layers are required. These layers may contribute to an increased inhomogeneity (e.g. through thickness variation) and wave reflection from impedance mismatch.

The main aim of the current work has been to build prototypes for adhesive-free highfrequency ultrasonic focused transducers and to investigate their suitability as transducers for high frequency ultrasonic imaging. In particular, we have used the transducer to image the surface of flexible electronic circuits (Kapton circuits). Flexible circuits are typically covered with a thin dielectric cover layer, and the suggested surface imaging can be used to detect defects (e.g. scratches and holes) in this cover layer. The suggested transducers will have advantages in terms of low production cost and simple expansion to multi-element (array) transducers. The ability of make array transducers has been explored by producing four active focused transducer elements on a high performance PEI substrate.

In the proposed transducer layout, focusing of the ultrasonic beam is produced by milling out a spherical cavity for each transducer element in the PEI substrate as shown by the side view 
illustration in Fig. 1 (a). The PEI polymer is known to have a very good thermal stability, good impedance match to the PVDF copolymer and very low acoustic attenuation. ${ }^{6}$ For each PEI substrate with the dimension of $30 \times 30 \mathrm{~mm}^{2}$ size and $0.85 \mathrm{~mm}$ in thickness, four spherical cavities were initially engraved using a milling drill. The tip of the milling drill were drilled $0.65 \mathrm{~mm}$ into the material leaving $0.2 \mathrm{~mm}$ space to the opposite flat PEI surface. The outer periphery diameter of the spherical cavities was $2 \mathrm{~mm}$ as shown Fig. 1 (a). A thin layer of silver was sputtered on the flat side of the substrate pointing away from the cavity. The sputtering was done through a high-resolution metal mask containing four $1.6 \mathrm{~mm}$ diameter holes for producing circular active apertures co-centered with the PEI cavities. Each of these holes was connected to two elongated rectangular openings terminated by circular holes to make two electrode arms with circular connection points.

Then, after making the lower electrode pattern, dissolved P(VDF-TrFE) (77:23, molar ratio) was spin coated on top of the electrode and annealed at a temperature of $130^{\circ} \mathrm{C}$ for 8 hours to increase the crystallinity. Finally, the upper electrode with a silver target was sputtered on the top of the P(VDF-TrFE) through another patterned metal mask containing four circular hole $1.8 \mathrm{~mm}$ diameter and one electrode arm, which completes the assembly process. Here, the thickness of both the upper and lower electrode were estimated to be around $80 \mathrm{~nm}$ using a nano-profiler (KLA Tencor P6), while the annealed PVDF copolymer film thickness was estimated to $12 \mu \mathrm{m}$ using the same instrument. An image of the focused transducer panel with four circular apertures and 12 connection points is shown in Fig. 1(b). Here, for each aperture one electrode line denoted as sender $T_{x}$ and two electrode lines denoted as $R_{x 1}$ and $\mathrm{R}_{\mathrm{x} 2}$ for receiver.

In order to make the $\mathrm{P}(\mathrm{VDF}-\mathrm{TrFE})$ layers piezoelectric, they were polarized at room temperature with a high voltage $\mathrm{AC}$ source connected to the lower electrodes $\mathrm{R}_{\mathrm{x} 1}$ and $\mathrm{R}_{\mathrm{x} 2}$, while the upper ones $\mathrm{T}_{\mathrm{x}}$ were grounded.

To drive the transducers a RF amplifier (Electronics \& Innovation 411LA) with $40 \mathrm{~dB}$ gain was applied. The input to this amplifier was a broad-banded Ricker wavelet $\left(2^{\text {nd }}\right.$ derivative of a Gaussian) obtained from an arbitrary waveform generator (Agilent 81150A). This amplified pulse was fed into the upper transducer electrode generates a pressure wave that propagates through the water medium and becomes reflected from a glass plate, which its surface located at the focal point of the transducer. Then, the current induced from this reflection is then pick up by the lower transducer electrode, and amplified by a trans-impedance amplifier (FEMTO DHPCA-100 with $10^{4}$ V/A set as the converting factor) on receiver side. The output voltage 
from this amplifier is finally digitized in an oscilloscope (Agilent 3024A).

Fig. 2 (a) shows the time-domain acoustical reflection induced by the glass reflector for one of the transducer elements. The focal distance where the maximum amplitude is obtained around $2.6 \mathrm{~mm}$ matches well with the designed spherical cavities. Here, it should be noted that the focal point of the transducer depends on the depth and the diameter of spherical curvature which were also calculated from COMSOL software. The corresponding frequency spectra in $\mathrm{dB}$ scale, is shown in the Fig. 2(b). The frequency response can be estimated from the ratio between the output spectra from the glass reflector and input spectra of a Ricker wavelet. This gives a center frequency around $48.5 \mathrm{MHz}$, with a lower and upper $-6 \mathrm{~dB}$ bandwidths around 25 and $76.5 \mathrm{MHz}$, yielding a bandwidth of $94.2 \%$. It should be noticed that several factors might influence the measured acoustic responses, like the bandwidth limitation for the current amplifier, wave effects (e.g. diffraction and attenuation), and surface roughness of the imposed materials (PVDF and PEI).

The transducer prototypes were used to image flexible circuits made from polyimide (PI) or Kapton with thickness around $84 \mu \mathrm{m}$ producing images as shown in Fig. 3(a), (b) and (c). These circuits contain a layered structure of patterned copper electrode and PI embedded in a two-side solder mask. The transducer substrate were mounted in a three-dimensional (3D) scanning tank with one of the four transducer elements exited by the broad-banded pulse, and moved in a two-dimensional (2D) plane parallel to the substrate plane (denoted as the xyplane). The total scan area was $2.2 \mathrm{~mm} \times 2.2 \mathrm{~mm}$ with a $20 \mu \mathrm{m}$ step length in both directions. The transducer was focused on the top layer of the solder mask cover, and the voltage amplitudes for each step or pixel, were plotted to create 2D images. Here, Fig. 3(a) shows the ultrasonic image generated from a flexible circuit without any defects within the scanning areas, whereas the images in Fig. 3(b) and (c) revile artificial defects like scratches and cuts. The corresponding optical images for Figs. 3(a), (b), and (c) taken with slightly shifted frames, are shown in Figs. 3(d), (e) and (f), respectively. Here, all 2D images showed very detailed variations with the expected resolution, with defects in the solder mask cover clearly visible in Fig. (e) and (f). The features of these defects look rather similar in the ultrasonic and optical images, and can therefore easily be identified.

To get a better understanding of the origin of the amplitude images, the reflection time series obtained at the four different spatial locations have been explored in details in Fig. 4. These examples are typical for the back scattering obtained from different location marked in Fig. 
3(b). The legend in the figure refers to the acoustic measurement position marked in Fig. 3 (b). The time series in Fig. 4(a) and 4(b) show the reflection from a non-scratched cover layer without and with under laying copper, respectively. The Fig. 4(c) and Fig. 4(d) show the acoustic reflection from a scratched cover layer without and with copper under. As expected, the strongest backscattering is observed when the transducer was focused toward an under laying copper electrodes i.e. [Fig. 4 (b)]. The highest amplitudes is also observed with some delay with respect to the initial wave feature, which is due to the thin cover layer in front of the copper. However, even if the cover layer delays the signal, it is probably too thin to be resolved for the applied frequencies. Moreover, the amplitudes of the reflection decreased sharply when the transducer was focused on the scratched area.

The present study has shown that it is possible to produce reliable polymer focused transducers from a layer-by-layer deposition method including milled spherical cavies in a PEI polymer substrate. The proposed method which process $\mathrm{P}(\mathrm{VDF}-\mathrm{TrFE})$ from the fluid phase, is adhesive-free in the sense that it does not require any additional adhesive layers for material binding. The transducer center frequency was estimated to $48.5 \mathrm{MHz}$ with $94.2 \%$ bandwidth. The two-dimensional scanning showed very detailed surface variations with the expected resolution, with defects in the solder mask clearly visible in acoustic scanning images. It is also believed that the proposed high frequency transducers can be used for measuring effects normally hidden in optical images. Examples here are surface roughness under optically opaque solder mask, copper roughness on the side facing the laminate, and internal delamination close to the surface.

\section{Acknowledgement}

This work was supported by The Research Council of Norway through the project "Subsea sensors". 


\section{References}

1) M. Robert, G. Molingou, K. Snook, J. Cannata, and K. K. Shung, J. Appl. Phys. 96, 252 (2004).

2) J. S. Jeong, and K. K. Shung, Ultrason. 53, 455 (2013).

3) L. F. Brown, R. L. Carlson, and J. M. Sempsort, Proc. of 1997 IEEE Ultrason. Symp. 1997, p. 1725 .

4) F. S. Foster, K. A. Harasiewicz, and M. D. Sherar, IEEE Trans. on Ultrason. Ferroelectr. Freq. Control. 47, 1363 (2000).

5) S. Wagle, A. Decharat, P. Bodö and F. Melandsø: Appl. Phys. Lett. 103, 262902 (2013).

6) S. Wagle, A. Decharat, A. Habib, B. S. Ahluwalia and F. Melandsø: Jpn. J. Appl. Phys. 55, 07KE11 (2016).

7) F. Melands $\varnothing$, S. Wagle, A. Decharat, A. Habib and B.S. Ahluwalia: Jpn. J. Appl. Phys. 55, 07KB07 (2016).

8) A. Decharat, S. Wagle and F. Melandsø: Jpn. J. Appl. Phys. 53 (2014) 05HB16

9) A. Habib, A. Shelke, M. Vogel, U. Pietsch, X. Jiang and T. Kundu: Ultrasonics. 52, 989 (2012).

10) A. Habib, A. Shelke, M. Vogel, S. Brand, X. Jiang, U. Pietsch, S. Banerjee and T. Kundu, Acta Acust united Ac. 101, 675 (2015).

11) Y. C. Lee, Jpn. J. Appl. Phys. 40, 359 (2001).

12) W. Zou, S. Holland, K.Y. Kim and W. Sachse, Ultrasonics. 41, 157 (2003).

13) S. Smolorz and W. Grill, Res. Nondestr. Eval. 7, 195 (1996). 


\section{List of figures}

Fig. 1 (Color online) (a) illustration of focal point, (b) Image of the final focused transducer containing 4 circular apertures

Fig. 2 (a) (Color online) Time domain acoustic reflection of the focused transducer from the glass plate. (b) Frequency spectra in a dB corresponding to (a)

Fig. 3 (a) (Color online) Acoustic images of the flexible circuit without defects and (b) \& (c) with defects (scratches), (d), (e) and (f) corresponding optical images.

Fig. 4 (Color online) Time domain acoustic reflection of the focused transducer from the PI circuit (a) at solder mask cover layer without copper electrode under lying (b) with copper electrode under lying, (c) at scratched surface without copper electrode under lying, (d) at scratched surface with copper electrode under lying. Legends in the figure refer to the acoustic measurement position marked in Fig. 3 (b). 